\title{
IOT Based Coal Mine Safety Monitoring and Alerting System
}

\author{
Prof. A. H. Ansari, Karishma Shaikh, Pooja Kadu, Nikam Rishikesh \\ Department of Electronics and Telecommunication Engineering, Pravara Rural Engg. College, Loni, India
}

\begin{abstract}
Article Info

Volume 8, Issue 3

Page Number: 404-410

Publication Issue :

May-June-2021

\section{Article History}

Accepted : 10 June 2021

Published: 17 June 2021

Safety is the most vital part of any type of industry. In the mining industry safety and security is a fundamental aspect of all. To avoid any types of accidents mining industry follows some basic precautions. Still accidents take place in underground mines due to rise in temperature, increased water level, and methane gas leakage. Here we provide safety to worker. When worker in danger he can press panic switch inform security. To enhance safety in underground mines, a reliable communication system must be established between workers in underground mines and fixed ground mine system. The communication network must not be interrupted at any moment and at any condition. A cost effective zigbee based wireless mine supervising system with early-warning intelligence is proposed in this project. Worker status can be monitor over IOT.

Keywords - PIC Microcontroller ,wifi / GSM moule ,zigbee ,temperature sensor, gas sensor, water level sensor
\end{abstract}

\section{INTRODUCTION}

Mines are the world's most dangerous place to work because in the mines, explosion often happens and thousand people are dying. And a recent report states that in such mine accidents an average of around 12,000 people have died. Coal is a nonsustainable origin that cannot be widely replaced by humans, there are several mishaps of coalmines occurring in the mines, and the diggers are putting their lives at risk, by working in the coal mines, even once in a while they end up losing their lives in the coal mines that are an unfortunate part. Mainly such mishaps happen as a direct result of the old equipment and wired devices, resulting in the end, mishandling, spillage of the noxious gases in the coal mines, pose tremendous hazards to the excavators inside the coal mines. So we've designed the coalmine protection system to stay away from this problem. We tackled the issues in our research by testing each of the information collected by the sensors, we use and finishing the analysis using the Thinger system. Controlling can be done automatically or manually.

\section{Literature Survey}

Yongping $\mathrm{Wu}$ and Guo Feng implement coal mine monitoring using the Bluetooth wireless transmission system. As a standard of unified global short-range wireless communication, Bluetooth technology is to 
establish a common low-power, low-cost wireless air interface and controlling software opening system. This paper describes the development background, technical features and the structure of the protocol stack of Bluetooth technology, and proposed the solutions of the Bluetooth host controller interface (HCI) wireless communication for the complexity of its development [1].

Zhenzhen Sun proposed DCS Coal Mine Monitoring System Based on RS485 Bus, RS485 bus structure supports multi-point and two-way communication. So, this type of monitoring system can be developed using common 8-bit microcontrollers. It has the advantages of simple circuit structure and low costs. However, due to the adoption of master-slave structure network, it is difficult to guarantee the reliability of the network structure. Furthermore, the data transmission distance is limited with a poor realtime performance [2-3].

Jingjiang Song, Yingli Zhu proposed automatic monitoring system for coal mine safety based on wireless sensor network. This system design monitoring for coal mine safety constructed by MSP430F and nRF2401. The sensor groups of the system intensively monitor temperature, humidity and other parameters in the underground mine, parameters measured are sent to wireless communication module by the microcontroller. The collected information is sent to long-distance monitoring center by cable [4]. The problem of this implementation is that hardware is placed inside the coal mines, when a natural calamity or a roof fall occurred, the system is damage. So the reliability and long life of conventional communication system is poor. Due to the harsh environment inside the mine, the installation and maintenance of the system is very difficult. The another problem is that the working condition of coal mine is very noisy and if the distance of miner and system is long, miner not get proper message.

Y ogendra S Dohare and Tanmoy Maity design surveillance and safety system for underground coal mines based on Low Power WSN. In this system a low power, cost-effective, and Zigbee protocol based wireless sensor network that provides an intelligent surveillance and safety system for underground coal mines. The system consists of wireless connection of several nodes. This network can be easily placed in underground mines and it provides an effectively surveillance and safety system for underground coal miners. Especially, it provides the real-time data communication between miners and surface control room through highly secure, reliable wireless sensor nodes [5]. This system is placed in mine so problem is created when miner are not in range of the system. This system only monitor environmental condition of underground mine but not monitor the health condition of miner.

\section{PROPOSED SYSTEM}

\section{Block Diagram Description:}

This monitoring system contains several components like boards (PIC board, Xbee module and Zigbee USB interfacing board), LCD (Liquid crystal display), different sensors and other small electronic components. This chapter gives a detailed review of each of this part along with its working principle.

In this proposed system the coal mine safety systems are fixed with gas sensor modules, temperature sensor, water level sensor and relays. We integrate all the sensors to the controller. First we need to create an account in the ThingSpeak platform. In this system we mainly have monitoring and controlling systems. In monitoring system we monitor all the data from different sensors. Gas sensor detects the gas in the 
coal mine environment. If the gas level exceeds the normal level then the buzzer gets high so that the mine workers get notified. These sensor values are continuously uploaded to the cloud . for analysis and also for further use. The temperature and water level values are also monitored inside the coalmine and send data control unit through zigbee. The proposed system block diagram shown in fig

Control Unit:

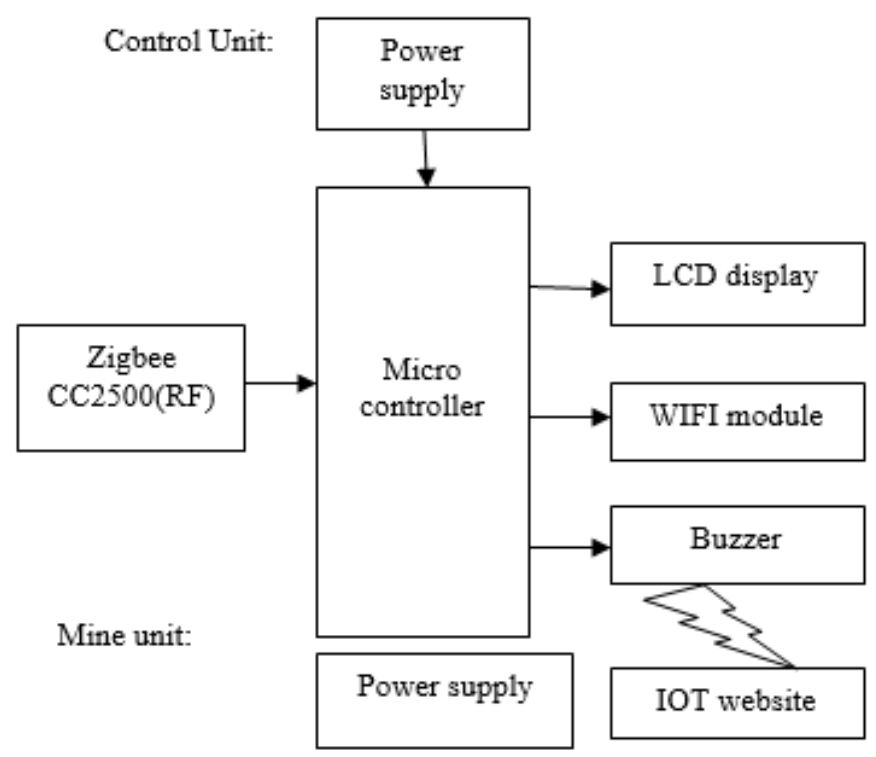

Fig.1.Block Diagram of the System

This monitoring system contains several components like boards (PIC board, Xbee module and Zigbee USB interfacing board), LCD (Liquid crystal display), different sensors and other small electronic components.

\section{1-PIC 18F4520}

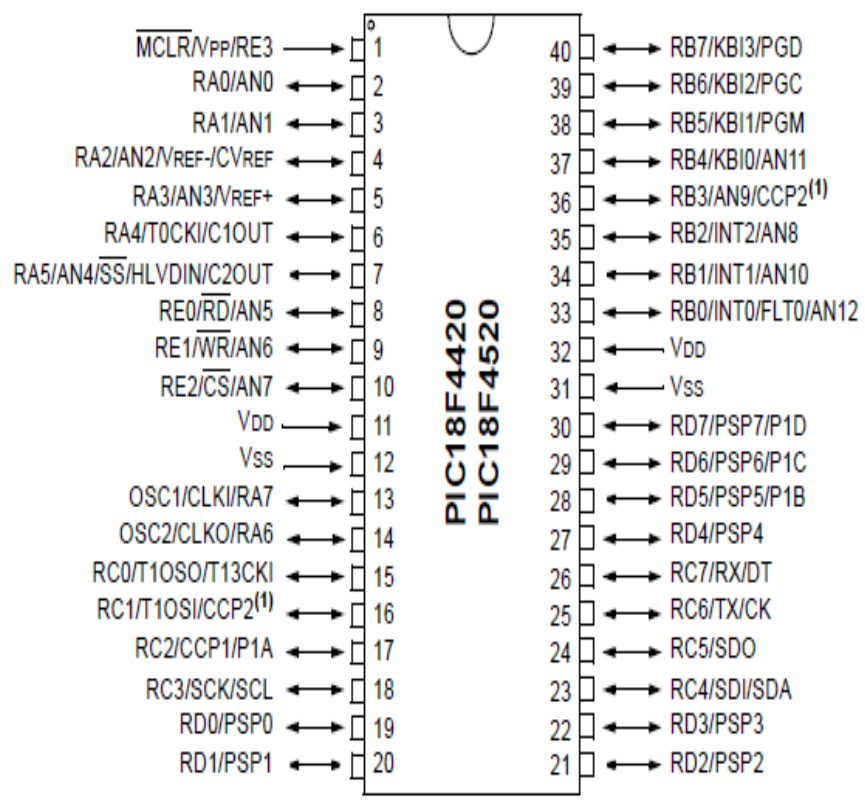

Fig.2. Pin Diagram of PIC 18F4520

Operating frequency $20 \mathrm{MHz} 40$ pins

\section{2 -ZIGBEE}

The CC2500 is a low-cost $2.4 \mathrm{GHz}$ transceiver designed for very low-power wireless applications. The circuit is intended for the $2400-2483.5 \mathrm{MHz}$ ISM (Industrial, Scientific and Medical) and SRD (Short Range Device) frequency band. The RF transceiver is integrated with a highly configurable baseband modem. The modem supports various modulation formats and has a configurable data rate upto $500 \mathrm{k}$ Baud.

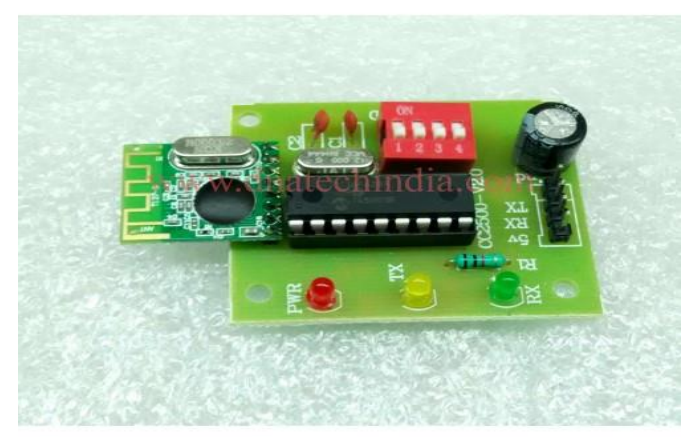

Fig-3 Zigbee 


\section{3- LM35 TEMPERATURE SENSOR}

Outputs $10 \mathrm{mV}$ per Degree that can also be read directly on multimeter or read in to microcontroller. For example at 30 degree celcius it will output $300 \mathrm{mV}$ at linear scale. The LM35 series are precision integrated-circuit temperature sensors, whose output voltage is linearly proportional to the Celsius (Centigrade) temperature. The LM35 thus has an advantage over linear temperature sensors calibrated in ${ }^{\circ}$ Kelvin, as the user is not required to subtract a large constant voltage from its output to obtain 25 convenient Centigrade scaling.
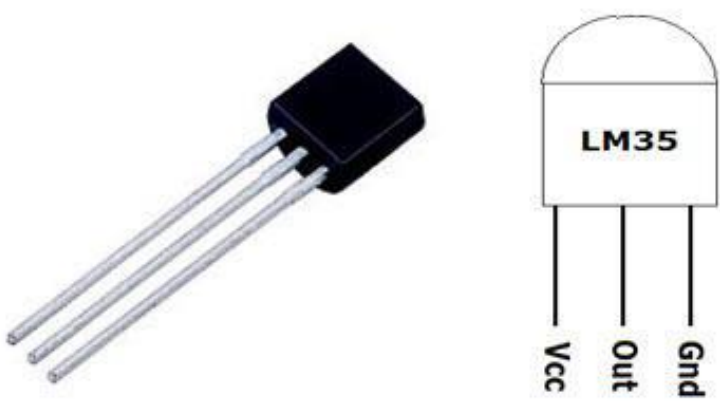

Fig.4-. LM35 Temperature Sensor

\section{4 -16*2 LCD DISPLAY}

LCD (Liquid Crystal Display)) screen is an electronic display module and find a wide range of applications. A $16 \times 2$ LCD display is very basic module and is very commonly used in various devices and circuits. These modules are preferred over seven segments and other multi segment LEDs. The reasons being: LCDs are economical; easily programmable; have no limitation of displaying special \& even custom characters (unlike in seven segments), animations and so on. A 16x2 LCD means it can display 16 characters per line and there are 2 such lines. In this LCD each character is displayed in 5x7 pixel matrix. This LCD has two registers, namely, Command and Data.

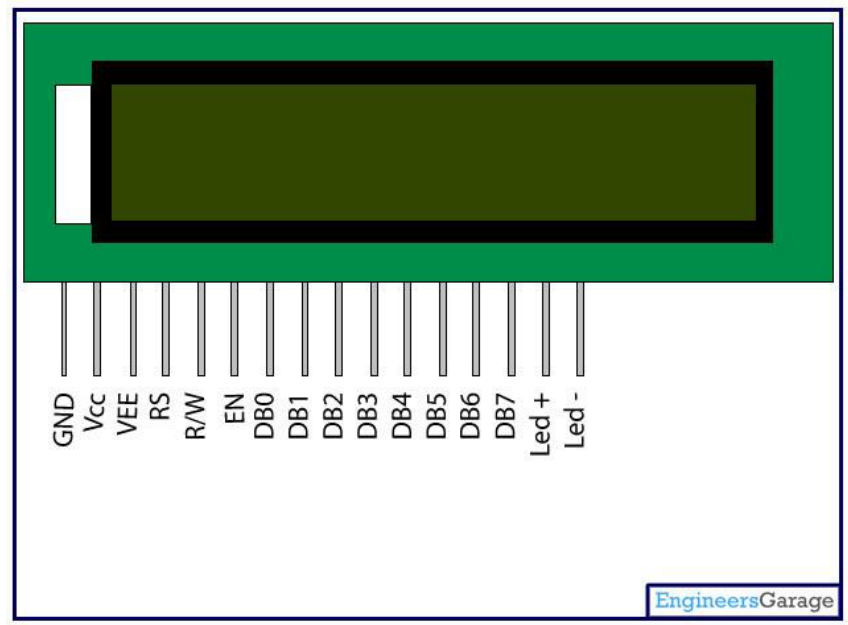

Fig.5 -16*2 Lcd Display

\section{5 -GSM MODULE}

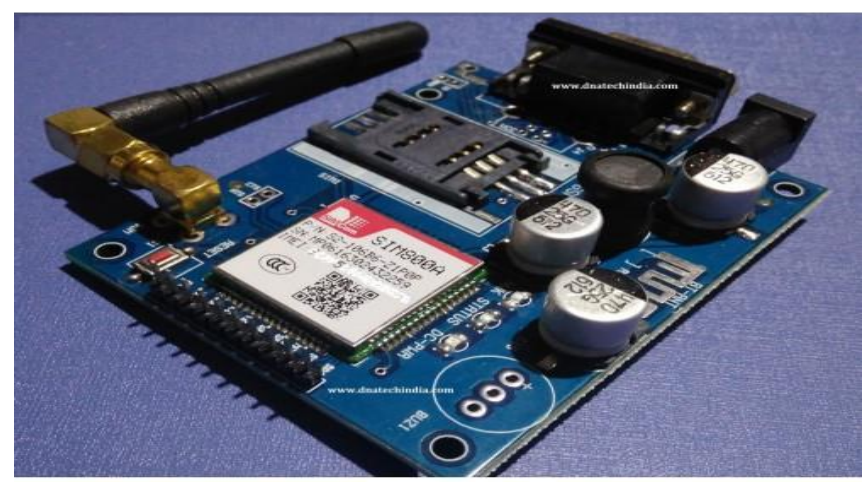

Fig-6 GSM Module

This GSM modem has a SIM800A chip and RS232 interface while enables easy connection with the computer or laptop using the USB to Serial connector or to the microcontroller using the RS232 to TTL converter. Once you connect the SIM800 28 modem using the USB to RS232 connector, you need to find the correct COM port from the Device Manger of the USB to Serial Adapter. Then you can open Putty or any other terminal software and open an connection to that COM port at 9600 baud rate, which is the default baud rate of this modem.Once a serial connection is open through the computer or your microcontroller you can start sending the AT commands. When you send AT commands for example: "AT $\backslash \mathrm{r}$ " you should receive back a reply from the SIM800 modem saying "OK" or other response depending on the command send. 


\section{6- PIEZOELECTRIC BUZZER}

Features

o sealed: yes

o operating power: $3-6 \mathrm{~V}$ DC / $25 \mathrm{~mA}$

o extremely compact, ultrathin construction

o no electrical noise

o low current consumption yet high sound pressure level

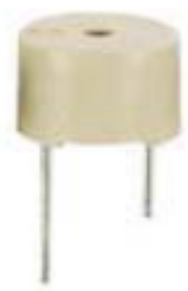

Fig.11 Piezoelectric Buzzer

\section{7 -MQ- GAS SENSOR}

Using a MQ sensor it detect a gas is very easy. You can either use the digital pin or the analog pin to accomplish this. Simply power the module with $5 \mathrm{~V}$ and you should notice the power LED on the module to glow and when no gas it detected the output LED will remain turned off meaning the digital output pin will be $0 \mathrm{~V}$. Remember that these sensors have to be kept on for pre-heating time (mentioned in features above) before you can actually work with it. Now, introduce the sensor to the gas you want to detect and you should see the output LED to go high along with the digital pin, if not use the potentiometer until the output gets high. Now every time your sensor gets introduced to this gas at this particular concentration the digital pin will go high $(5 \mathrm{~V})$ else will remain low $(0 \mathrm{~V})$. You can also use the analog pin to achieve the same thing. Read the analog values $(0-5 \mathrm{~V})$ using a microcontroller, this value will be directly proportional to the concentration of the gas to which the sensor detects. You can experiment with this values and check how the sensor reacts to different concentration of gas and develop your program accordingly.

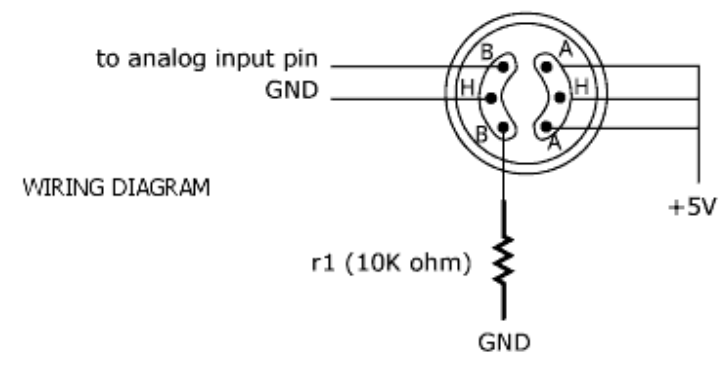

Fig.12 Diagram Of Gas Sensor

\section{8 - WATER LEVEL SENSOR}

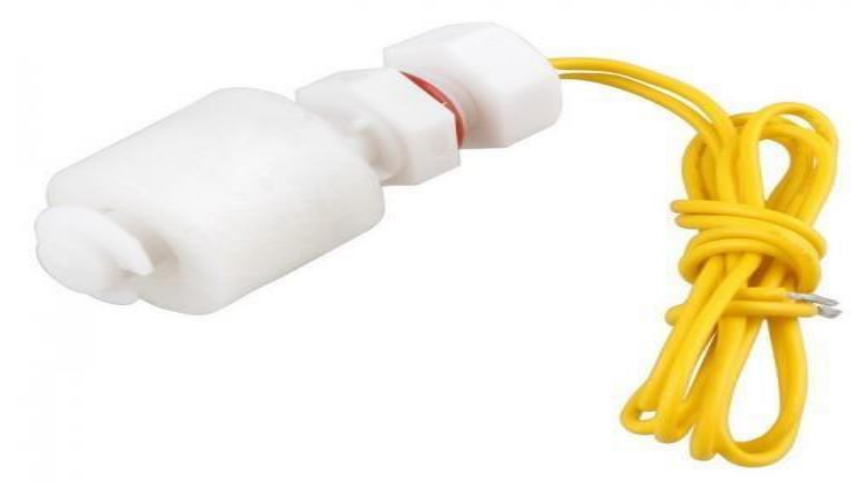

Fig.13- Water Level Sensor

A float switch is a device used to sense the level of liquid within a tank, it may actuate a pump, an indicator, an alarm, or other device. A float switch is a device used to sense the level of liquid within a tank. The switch may actuate a pump, an indicator, an alarm, or other device. Use them with hydroponics, saltwater tank, freshwater tank, gardening, aquariums for power head control, pet bowls, fish tanks, filtration, heating, pumps, ponds, basement alarms, boats, air condition drain pans, pressure washers, carpet cleaning mach, reef aquarium, fluid control, ice machines, coffee pots, marine, automotive, automobiles, tropical fish tanks, evaporator coils, condensation line, in relays, or what ever your project may be. It can be easily converted from normally open to normally close by inverting the float. 


\section{ADVANTAGES:}

- Cost is less

- Provide wireless connection security.

- Improved services in coal mine.

\section{AP PLICATIONS}

- For Underground Mine System

\section{RESUL:}

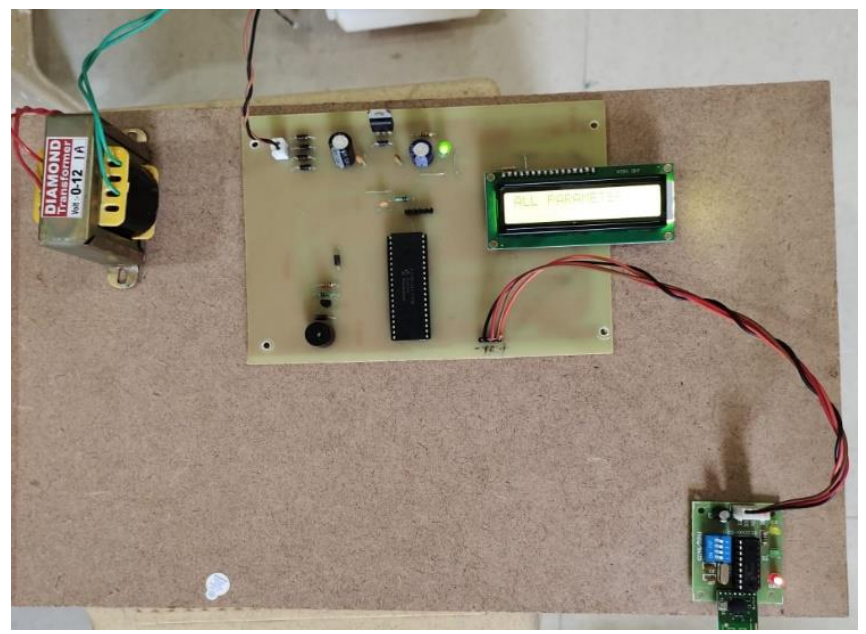

Fig.14 Control unit

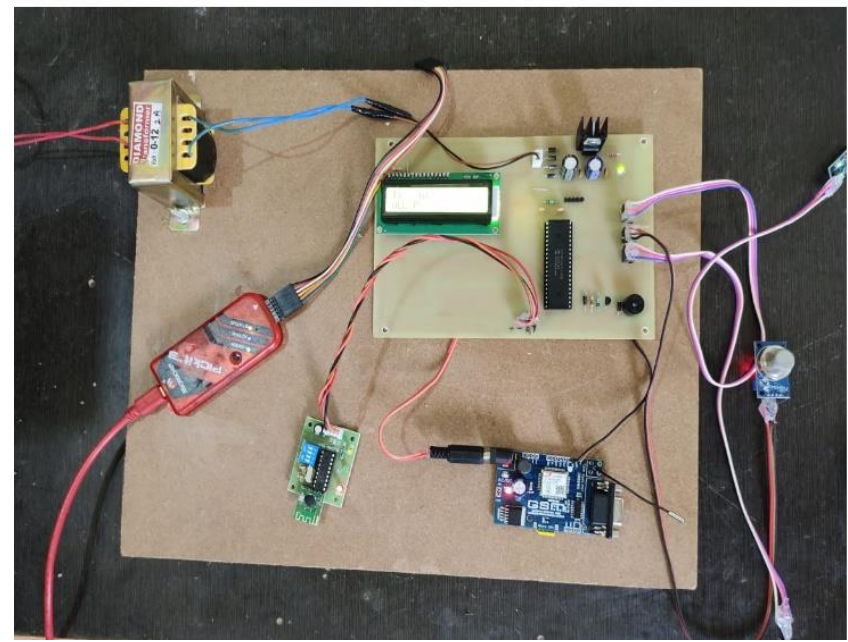

Fig.15 Mine unit

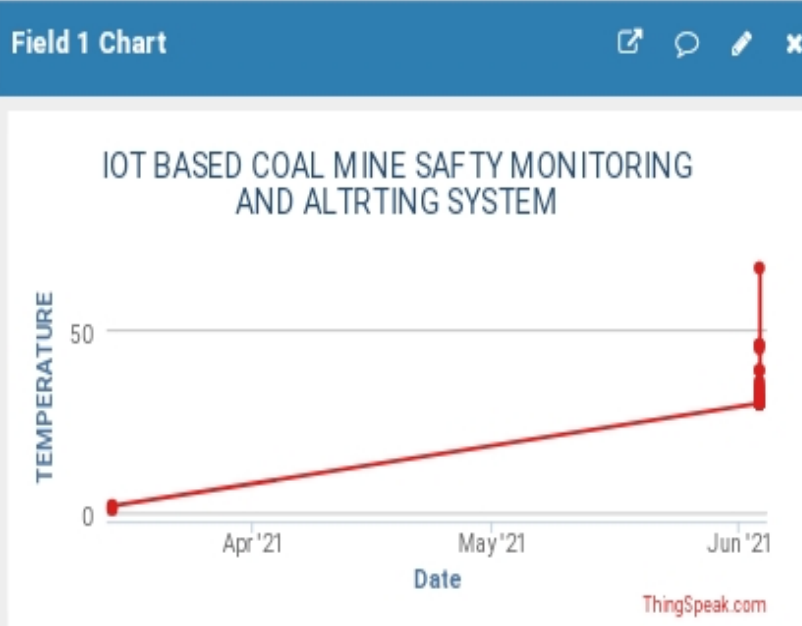

Field 2 chart

$\square 0 / x$

IOT BASED COAL MINE SAFTY MONITORING AND ALTRTING SYSTEM

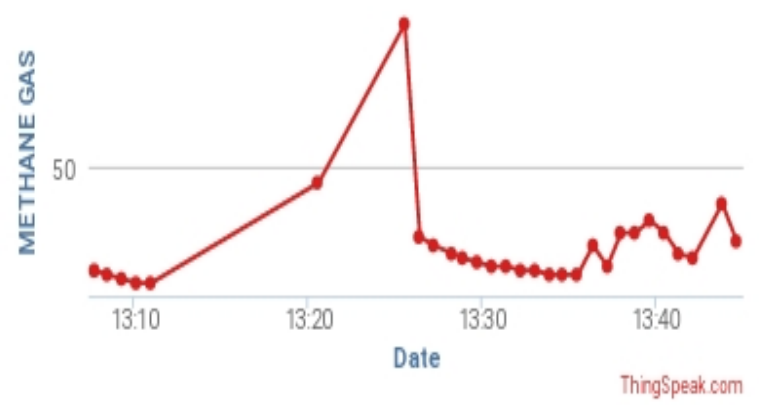

Field 3 Chart

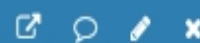

IOT BASED COAL MINE SAFTY MONITORING AND ALTRTING SYSTEM

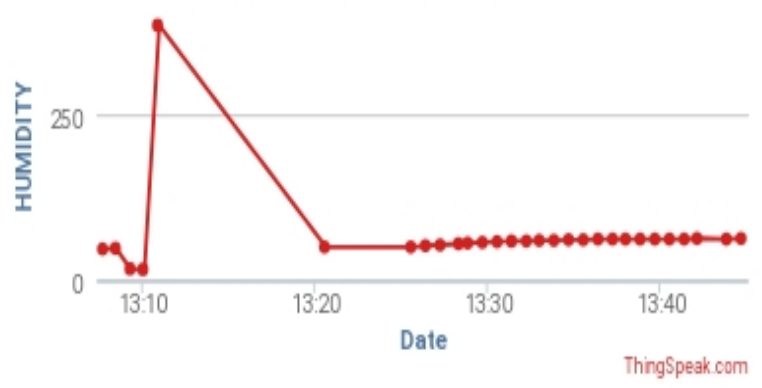

Fig.16 Output on IOT website 
From above graph it is seen that whenever the parameters exceeds the preset limit i.e. when temperature is more than $50^{\circ} \mathrm{C}$, gas concentration is more than 50 and humidity is more than 250 then the designed system will show alert message on lcd display and buzzer will sound. Also, IOT website gives this information to distant person hence it helps to prevent dangerous hazard .

\section{REFERENCES}

[1]. Yongping $\mathrm{Wu}$ and Guo Feng, "The study on coal mine monitoring using the Bluetooth wireless transmission system", 2014 IEEE Workshop on Electronics, Computer and Applications, pp. 1016-1018, 2014.

[2]. Xiaolong Feng, Jiansheng Qian, Zhenzhen Sun, Xing Wang, "Wireless Mobile Monitoring System for Tram Rail Transport in Underground Coal Mine Based on WMN," cason, pp.452-455, 2010 International Conference on Computational Aspects of Social Networks, 2010.

[3]. Yi-ming Tian, You-rui Huang, Yi-qing Huang, "Intelligent Information Processing of WSN Based on Vague Sets Theory and Applied in Control of Coal Mine Monitoring,"cccm, vol. 2, pp.649-652, 2008 ISECS International Colloquium on Computing, Communication, Control, and Management, 2008.

[4]. Jingjiang Song, Yingli Zhu and Fuzhou DongK, "automatic monitoring system for coal mine safety based on wireless sensor network", IEEE Radio Science and Wireless Technology Conference, pp.933-936, 2011.

[5]. Yogendra S Dohare and Tanmoy Maity, "surveillance and safety system for underground coal mines based on Low Power WSN", IEEE, pp.116-119, 2014.

[6]. Valdo Henriques and Reza Malekian, " Mine safety system using wireless sensor network", IEEE, pp. 1-12, 2016.
[7]. Huping $\mathrm{Xu}$, Feng Li, Yancheng Ma, A ZigBeebased miner Localization System', IEEE, 2012. 8 Shuo pang, Ricardo Trujillo, Indoor Localization Using Ultrasonic Time Difference of Arrival', IEEE, 2013.

[8]. Yongping Wu, Guo Feng, Zhang Meng, The Study on Coal Mine Using the Bluetooth Wireless Transmission',IEEE, 2014.

[9]. Yuping Zhang, Yinghui Zhang, Chen Li2, Research of Short Distance Wireless Communication Technology in the Mine Underground',IEEE, 2014.

[10]. Manash Jyoti Deka, Jetendra Joshi, Nishchay Sinha, Aman Tyagi, Apoorv Kushal Avijit Jain, Indoor and Outdoor Position Identification Using RFID', IEEE, 2016.

[11]. Mengda Wang, Bing Xue, Wei Wang, Junjie Yang, The Design of multi-User Indoor UWB Localization System',IEEE, 2017.

[12]. Nisha Dube1, Prof. K.S.Ingle 2 PG Student, Dept. of ECE “Intelligent Mining: A Monitoring and Security System for Coal Mine Workers",International Journal of Advanced Research in Electrical, Electronics and Instrumentation Engineering (An ISO 3297: 2007 Certified Organization) Vol. 5, Issue 1, January 2016.

\section{Cite this article as :}

Prof. A. H. Ansari, Karishma Shaikh, Pooja Kadu, Nikam Rishikesh, "IOT Based Coal Mine Safety Monitoring and Alerting System", International Journal of Scientific Research in Science, Engineering and Technology (IJSRSET), Online ISSN : 2394-4099, Print ISSN : 2395-1990, Volume 8 Issue 3, pp. 404-410, May-June 2021. Available at doi : https://doi.org/10.32628/IJSRSET2183188 Journal URL : https://ijsrset.com/IJSRSET2183188 